\title{
An income over feed cost nutritional grouping strategy
}

\author{
Y. Wu, D. Liang, R. D. Shaver, and V. E. Cabrera* \\ Department of Dairy Science, University of Wisconsin-Madison 53705
}

\section{ABSTRACT}

This study introduces a new nutritional grouping method, OptiGroup, which maximizes milk income over feed cost (IOFC) using a mixed-integer nonlinear programming optimization algorithm. Analyses compared the OptiGroup with the cluster method, the current state-of-the-art nutritional grouping technique. Analyses were performed using cow-level data from 7 Wisconsin dairy farms. Consistently, the OptiGroup and the cluster were constrained to group cows simultaneously into 2 (low and high nutrient requirements) and 3 (low, medium, and high nutrient requirements) samesize groups. Each diet satisfied the net energy $\left(\mathrm{NE}_{\mathrm{L}}\right)$ and crude protein $(\mathrm{CP})$ requirements of approximately $83 \%$ of the cows in each group by using lead factors based on nutrient density. A control treatment (1-group scenario) was used as a baseline for comparisons. The IOFC, dietary nutrient densities $\left(\mathrm{NE}_{\mathrm{L}}\right.$ and $\left.\mathrm{CP}\right)$, and dry matter intake with both methods were computed and compared. The percentage of cows grouped differently and the percentages of primiparous cows and late-lactation ( $>200 \mathrm{~d}$ in milk) cows in each group were also analyzed. Results were as follows: (1) average extra IOFC of $\$ 8 /$ cow per yr (2-group) and $\$ 12 /$ cow per yr (3-group) by switching from cluster to OptiGroup method; (2) difference between dietary nutrient densities of the groups were reduced under OptiGroup method compared with cluster (i.e., $\mathrm{NE}_{\mathrm{L}}$ differences in 2 groups were $0.20 \mathrm{Mcal} / \mathrm{kg}$ for the cluster vs. 0.11 $\mathrm{Mcal} / \mathrm{kg}$ for OptiGroup); (3) dry matter intake decreased with increasing group numbers within a grouping method, and decreased from cluster to OptiGroup method with constant group numbers; (4) percentage of primiparous cows was greater in the low group of cluster and in the high group of OptiGroup; and (5) proportion of late-lactation cows tended to be greater in the low group in both grouping strategies. Results indicated that the OptiGroup performed economically

Received June 28, 2018.

Accepted January 6, 2019.

*Corresponding author: vcabrera@wisc.edu better than the cluster because of nutrient savings, even with high feed cost conditions. This study offers a new nutritional grouping paradigm, which could improve herd management on dairy farms. However, animal trials are needed to validate this new nutritional grouping method under farm conditions.

Key words: cluster, diet formulation, precision feeding, mixed-integer nonlinear programming

\section{INTRODUCTION}

Feeding a single TMR to all groups of lactating cows is a common practice on commercial dairy farms (Contreras-Govea et al., 2015). This practice is contrary to recent reports (Cabrera and Kalantari, 2016; Kalantari et al., 2016) where feeding multiple TMR within a herd to lactating cows grouped by nutritional status can improve milk income over feed costs (IOFC). Contreras-Govea et al. (2015) reported, from a multistate survey of dairy farmers, that the main barriers to multi-TMR nutritional grouping were the desire to minimize management complexity and labor costs, and the perception of reduced milk production when cows were moved from one nutrition group to another. The latter concern, however, is still not clear according to the study of Zwald and Shaver (2012), which regrouped $20 \%$ of cows and failed to observe a milk production decrease after the move. Further research on nutritional grouping is warranted and of major importance to the dairy industry, not only for the economic benefits mentioned, but also for potential reductions in excretion of nutrients into the environment by dairy herds (StPierre and Thraen, 1999).

After reviewing a series of studies to evaluate grouping cows by different criteria that included milk production, FCM, DairyMerit (a function of production and $\mathrm{BW}$ ), and cluster (using a $\mathrm{NE}_{\mathrm{L}}$ and $\mathrm{CP}$ density requirements for maintenance and production; McGilliard et al., 1983; Williams and Oltenacu, 1992; St-Pierre and Thraen 1999; Kalantari et al., 2016), Cabrera and Kalantari (2016) concluded that cluster was the most effective nutritional grouping strategy because it minimized the variation in nutrient requirements within a group and maximized the variation among groups, 
thereby allowing for more accurate ration formulation for more homogeneous groups. More accurate ration formulation means the differences of animal's nutrient requirements and dietary nutrient densities are smaller within a group, so that the formulated ration is closer to the cows' nutrient requirement. Also, economically, the cluster method resulted in greater IOFC per cow per yr than the other known grouping strategies (StPierre and Thraen, 1999). However, ration formulation in the cluster grouping strategy does not interact with the cows' allocation to each group to minimize the feed cost. Therefore, we postulate that there could be a more profitable way to nutritionally group lactating cows within a dairy herd. We hypothesized that maximizing IOFC across milking groups would result in greater herd-level IOFC under the same assumptions as the cluster grouping strategy. We propose a new nutritional grouping strategy that we call OptiGroup. The objective of this study is thus to develop and apply the OptiGroup and compare it with the current state-ofthe-art grouping strategy, cluster, regarding economics, nutritional performance, and herd demographics.

\section{MATERIALS AND METHODS}

\section{Dairy Herd Characteristics}

To group cows using either cluster or OptiGroup strategies, cows' DIM, DMI, BW, and milk productivity are needed. We collected cow-level DHI records of one test-day results from 7 Wisconsin dairy farms with Holstein cows in March 2017 (Table 1). These data included cow ID $(i)$, milk yield $\left(\mathrm{MY}_{i}\right)$, milk fat concentration $\left(\mathrm{FAT}_{i}\right)$, lactation number $\left(\mathrm{LAC}_{i}\right)$, and $\mathrm{DIM}$ $\left(\mathrm{DIM}_{i}\right)$ that were used to estimate individual cow daily DMI $\left(\mathrm{DMI}_{i}\right)$ according to NRC (2001; Eq. 1-2). The available data set did not include the current grouping strategy and the number of diets on each farm. However, the farms were assumed using 1-group ration for lactating cows because Wisconsin dairy farms group cows by lactation stage, lactation number, or health issues, not individual cows' nutritional requirements (Contreras-Govea et al., 2015).

We estimated the BW according to the Korver algorithm as a function of $\mathrm{LAC}_{i}$ and $\mathrm{DIM}_{i}$ (Korver et al., 1985) assuming linear BW change between nearby DIM. For primiparous cows, the BW was $545 \mathrm{~kg}$ at calving, $505 \mathrm{~kg}$ at $42 \mathrm{DIM}$, and $605 \mathrm{~kg}$ at $336 \mathrm{DIM}$; for multiparous cows, the BW was $670 \mathrm{~kg}$ at calving, 630 $\mathrm{kg}$ at 63 DIM, and $730 \mathrm{~kg}$ at 336 DIM (Carvalho et al., 2014; Holstein Association USA, 2018). Then, we calculated the daily cow-level $\mathrm{NE}_{\mathrm{L}}$ and $\mathrm{CP}$ requirements as a sum of the maintenance and lactation requirements with the following equations:

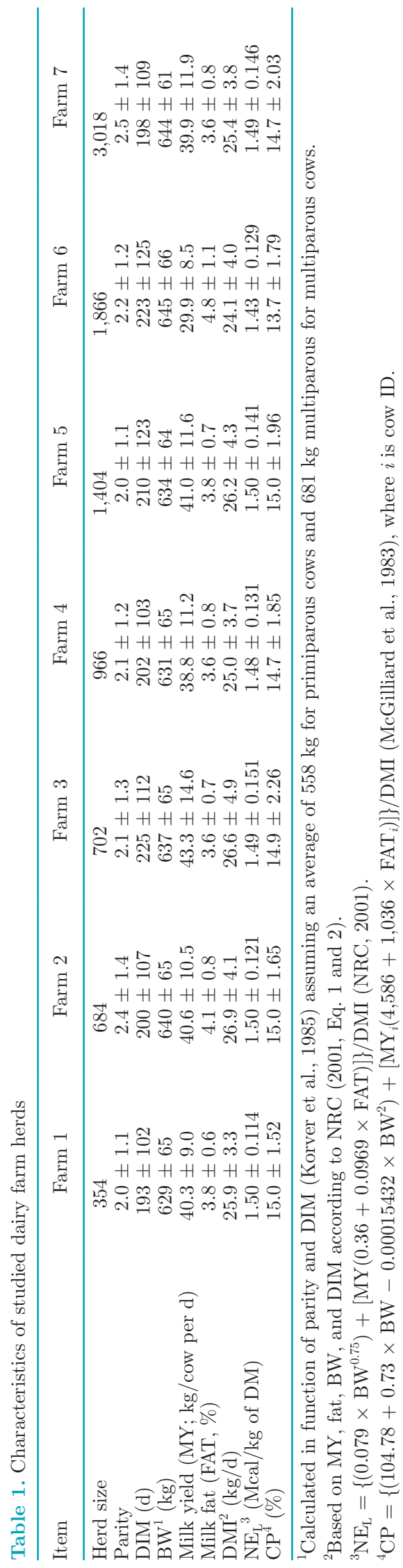

Journal of Dairy Science Vol. 102 No. 5, 2019 


$$
\begin{gathered}
\mathrm{NE}_{\mathrm{L} i}(\mathrm{Mcal} / \mathrm{d})=\left(0.079 \times \mathrm{BW}^{0.75}\right)+\left[\mathrm{MY}_{i}(0.36\right. \\
\left.\left.+0.0969 \times \mathrm{FAT}_{i}\right)\right](\mathrm{NRC}, 2001) \\
\mathrm{CP}_{i}(\mathrm{~g} / \mathrm{d})=[104.78+0.73 \times \mathrm{BW}-0.00015432 \\
\left.\times \mathrm{BW}^{2}\right]+\left[\mathrm{MY}_{i}\left(4,586+1,036 \times \mathrm{FAT}_{i}\right)\right]
\end{gathered}
$$

(McGilliard et al., 1983).

Finally, we divided these cow-level nutrient requirements by their corresponding $\mathrm{DMI}_{i}$ to express them as density (Mcal/kg for $\mathrm{NE}_{\mathrm{L}}$ and $\%$ for $\mathrm{CP}$ ).

Income over feed cost in this study was the farm-level difference between milk income and feed cost of the lactating cows. The feed cost was calculated based on the $\mathrm{NE}_{\mathrm{L}}$ and $\mathrm{CP}$ concentrations and not on the cost of feed ingredients. Prices of $\mathrm{NE}_{\mathrm{L}}$ and $\mathrm{CP}$ were collected from St-Pierre and Thraen (1999).

\section{The Cluster Grouping Strategy}

Cluster grouping strategy arranges cows with similar nutrient requirements into the same group (McGilliard et al., 1983). In this study, following Kalantari et al. (2016), the cluster grouping strategy was a 2-stage process. In the first stage, the model ranked all the cows from highest to lowest $\mathrm{NE}_{\mathrm{L}}$ requirement. Cows were arranged into multiple groups based on their ranking. In the second-stage, the model formulated diet for each group to maximize the farm-level IOFC. McGilliard et al. (1983) found that cluster grouping strategy allocates cows into more homogeneous groups of nutrient requirements. St-Pierre and Thraen (1999) demonstrated that cluster grouping strategy led to greater IOFC per cow per year than the other grouping criteria (i.e., FCM, solely $\mathrm{NE}_{\mathrm{L}}$, and solely $\mathrm{CP}$ ). In this study, we propose the OptiGroup as a new nutritional grouping strategy that could perform better than the cluster grouping strategy with respect to the resulting IOFC per cow per day. The statistical software $\mathrm{R}$ ( $\mathrm{R}$ Core Team, 2017, version 3.4.3) was used to compute this grouping process.

\section{The OptiGroup Model}

Different than the cluster grouping strategy, which arranges cows solely based on their nutrient requirement, the OptiGroup grouping strategy assigns cows to feeding groups interactively and to maximize group and herd IOFC. The OptiGroup grouping strategy is a mixed integer nonlinear optimization model (Bussieck et al., 1985) to maximize herd-level IOFC under nutritional grouping scenarios (i.e., 2 or 3 feeding groups). Different than the cluster method (McGilliard et al., 1983; St-Pierre and Thraen, 1999; Cabrera and Kalantari, 2016), OptiGroup assigned cows to groups interactively and recorded its corresponding IOFC. The objective function was to maximize the herd-level IOFC with the decision variable of each cow's allocation to a group $\left(g_{j i}\right)$, expressed as

maximum IOFC $=$
$\sum_{j=1}^{n}\left(P_{\text {milk }} \sum_{i=1}^{N_{j}} g_{j i} \mathrm{MY}_{i}-C_{\mathrm{NE}} \sum_{i=1}^{N_{j}} g_{j i} \mathrm{DMI}_{i} \mathrm{NE}_{\mathrm{L}, \text { diet } j}-C_{\mathrm{CP}} \sum_{i=1}^{N_{j}} g_{j i} \mathrm{DMI}_{i} \mathrm{CP}_{\text {diet } j}\right)$,

where $j=$ group number; $n=$ number of groups $(2$ or 3 ); $i=$ cow number; $N_{j}=$ number of cows in group $j$; $P_{\text {milk }}=$ price of milk; $g_{j i}=\operatorname{cow} i$ in group $j ; \mathrm{MY}_{i}=$ milk yield of cow $i ; C_{\mathrm{NE}}=$ cost of net energy nutrient by unit; $\mathrm{DMI}_{i}=$ estimated dry matter intake of cow $i$; $\mathrm{NE}_{\mathrm{L}, \text { diet } j}=$ net energy density in diet of group $j ; C_{\mathrm{CP}}=$ cost of crude protein by unit; and $\mathrm{CP}_{\text {diet } j}=$ crude protein percentage in diet of group $j$. Subject to the constraints: every cow belongs to a group, $\sum_{j=1}^{n} g_{j i}=1$; every cow only belongs to one group, $g_{j i} \in\{0,1\}$; groups are of the same size, $N_{1}=N_{2}$ for 2 groups and $N_{1}=N_{2}=N_{3}$ for 3 groups; groups diets are ranked by nutrient den-

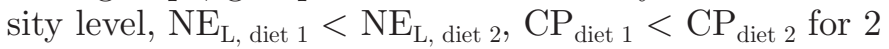
groups, and $\mathrm{NE}_{\mathrm{L} \text {, diet } 1}<\mathrm{NE}_{\mathrm{L} \text {, diet } 2}<\mathrm{NE}_{\mathrm{L} \text {, diet } 3}, \mathrm{CP}_{\text {diet } 1}$ $<\mathrm{CP}_{\text {diet } 2}<\mathrm{CP}_{\text {diet } 3}$ for 3 groups.

The model was developed using the general algebraic modeling system (Bussieck and Meeraus, 2004) with the BARON solver (Bussieck et al., 1985). Cow allocations in the cluster results were used as seed values to initialize the OptiGroup optimization process.

\section{OptiGroup vs. Cluster Comparisons}

We approached both strategies under similar conditions and assumptions. As normally assumed with cluster grouping strategy, we postulated that individual cow milk production will not change when supplied a diet within a range of its nutrient requirements when using a lead factor whether the cows were grouped according to cluster or OptiGroup. Since milk production did not change, maximum IOFC was achieved when minimum nutrient costs were attained. In both, OptiGroup and cluster, we set group sizes and lead factors to be exactly the same. We calculated the IOFC consistently with both methods using the same prices of $\$ 0.3 / \mathrm{kg}$ of milk, $\$ 0.07 /$ Mcal of net energy (NE), and $\$ 0.4 / \mathrm{kg}$ of CP (St-Pierre and Thraen, 1999). 
All the statistical analysis was conducted using $\mathrm{R}$. The effects of cluster and OptiGroup strategies were compared by one-way ANOVA according to a blocked design statistical model: $Y_{i j}=\mu+$ Treatment $_{i}+$ Farm $_{j}$, where $\mu$ is the grand mean, $i=1,2, \ldots, 5$ ( 1 is nongrouping, 2 to 5 are combinations of grouping strategies and number of groups), and $j=1, \ldots, 7$ (farms). The model considered the farm as the experimental unit and included the combination of grouping strategy and number of groups as a fixed effect and farm as a random effect. Least significant difference was employed to detect the group-level difference in the 3 -group scenario (high vs. medium group, high vs. low group, and medium vs. low group).

\section{Cows Grouped Differently}

We also calculated the number and proportion of cows grouped differently in OptiGroup with respect to cluster emphasizing the allocation of primiparous and late-lactation (DIM > 200) cows.

\section{Complexity of OptiGroup}

Mathematical programming with continuous and discrete variables and nonlinearities in the objective function and the constraints, such as the mixed integer nonlinear optimization model in OptiGroup, are difficult to solve (Arora et al., 1994). This difficulty increases even more when the number of decision variables is large (i.e., the number of cows in a herd; Grossmann, 1990). Ideally, diets for each group $\left(\mathrm{NE}_{\text {diet }}\right.$ and $\left.\mathrm{CP}_{\text {diet }}\right)$ should be interactively recalculated within the optimization algorithm as a percentile or variability of the density requirements of the assigned cows to that group. Trial tests indicated that this process would be unfeasible or unpractical (or both) when the number of cows in a group was larger than 90. This occurred even when solved using the most advanced computing system from the University of Wisconsin-Madison Center for High Throughput Computing. Therefore, we decided to follow a simplified approach to partially linearize the optimization problem and decrease its convergence complexity. This was achieved using a modified lead factor approach (Stallings and McGilliard, 1984) to calculate the $\mathrm{NE}_{\mathrm{L}}$ and $\mathrm{CP}$ to be fed to each group within the optimization algorithm.

\section{Lead Factor Calculation}

Lead factor is a multiplicative coefficient applied to the average nutrient requirement for diet formulation
(Cabrera and Kalantari, 2016). It is commonly used in diet formulation to adjust the diet nutrient content to exceed the average level in a group and ensure the higher milk productivity from cows with greater nutrient requirements. Diet formulation normally involves the use of lead factors to increase diet $\mathrm{NE}_{\mathrm{L}}$ and $\mathrm{CP}$ densities above the average cow requirements so as to not underfeed the higher producing cows in the group (Stallings and McGilliard, 1984). Stallings and McGilliard (1984) proposed using [(mean milk yield +1 $\mathrm{SD}) /$ mean milk yield] to generate lead factors for each group. We followed the same concept but used nutrient densities rather than milk yield. Therefore, the lead factors $(\alpha)$ were calculated as 1 SD above average NE and $\mathrm{CP}$ densities of studied group:

$$
\begin{aligned}
& \alpha_{\mathrm{NE}_{j}}= \\
& \left\{\left[\operatorname{mean}\left(g_{j i} \mathrm{NE}_{i}\right)+1 \mathrm{SD}\left(g_{j i} \mathrm{NE}_{i}\right)\right] / \operatorname{mean}\left(g_{j i} \mathrm{NE}_{i}\right)\right\}, \text { and } \\
& \alpha_{\mathrm{CP}_{j}}=\left\{\left[\operatorname{mean}\left(g_{j i} \mathrm{CP}_{i}\right)+1 \mathrm{SD}\left(g_{j i} \mathrm{CP}_{i}\right)\right] / \operatorname{mean}\left(g_{j i} \mathrm{CP}_{i}\right)\right\} .
\end{aligned}
$$

Lead factors were computed before solving the grouping optimizations to reduce computing complexities. We introduced a resampling technique to calculate the lead factors of studied herds and grouping scenarios. We generated randomly 6 subsamples of 90 cows each in every one of the 7 studied dairy herds. We grouped each subsample using both cluster (McGilliard et al., 1983) and OptiGroup. Then, we calculated $\mathrm{NE}_{\mathrm{L}}$ and $\mathrm{CP}$ lead factors for each subsample and grouping scenario. Finally, we averaged all the lead factors across all subsamples, grouping methods, and farms (Table 2 ) to generate the nutrient density lead factors. These lead factors were used to determine the diets inside the OptiGroup algorithm:

$$
\mathrm{NE}_{\text {diet }_{j}}=\operatorname{mean}\left(g_{j i} \mathrm{NE}_{i} \cdot \alpha_{\mathrm{NE}_{j}}\right)
$$

and

$$
\mathrm{CP}_{\text {diet }_{j}}=\operatorname{mean}\left(g_{j i} \mathrm{CP}_{i} \cdot \alpha_{\mathrm{CP}_{j}}\right)
$$

for each grouping scenario.

\section{Sensitivity Analysis on IOFC}

Sensitivity analysis was conducted to test the effect of economic factors $\left(\mathrm{NE}_{\mathrm{L}}\right.$ and $\mathrm{CP}$ prices) on the farmlevel IOFC with both cluster and OptiGroup strategies. 
Table 2. Estimated lead factors $(\alpha)$ based on nutrient requirements ${ }^{1}$

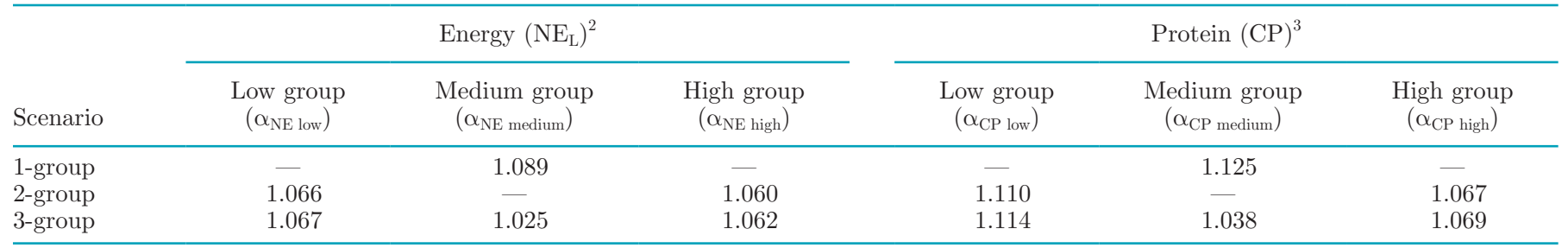

${ }^{1}$ Average of 6 subsamples of 90 cows each randomly generated in each one of the 7 studied Wisconsin dairy herds.

${ }^{2} \alpha_{\mathrm{NE}_{j}}=\left\{\left[\operatorname{mean}\left(g_{j i} \mathrm{NE}_{i}\right)+1 \mathrm{SD}\left(g_{j i} \mathrm{NE}_{i}\right)\right] /\right.$ mean $\left.\left(g_{j i} \mathrm{NE}_{i}\right)\right\}$, where $g_{j i}=$ cow $i$ in group $j, \mathrm{NE}_{i}=$ net energy requirement of cow $i$.

${ }^{3} \alpha_{\mathrm{CP}_{j}}=\left\{\left[\operatorname{mean}\left(g_{j i} \mathrm{CP}_{i}\right)+1 \mathrm{SD}\left(g_{j i} \mathrm{CP}_{i}\right)\right] /\right.$ mean $\left.\left(g_{j i} \mathrm{CP}_{i}\right)\right\}$, where $g_{j i}=$ cow $i$ in group $j, \mathrm{CP}_{i}=$ crude protein requirement of cow $i$.

The 2 scenarios of price change were $(1) \mathrm{NE}_{\mathrm{L}}$ price increase by $10 \%$ and (2) CP price increase by $10 \%$.

\section{RESULTS AND DISCUSSION}

\section{IOFC}

The average IOFC ( $\$$ /cow per yr) of the 7 studied farms was $\$ 2,596$ (range $\$ 1,772$ to $\$ 2,981$ ) without nutritional grouping (1-group; Table 3 ). The IOFC increased when cows were in multiple ration groups $(P<0.01$, Table 3$)$. Both grouping strategies increased IOFC with a greater number of feeding groups $(P<0.01$, Table 3$)$. With respect to 1 -group IOFC ( $\$ /$ cow per yr), there were positive differences of $\$ 40$ for 2 groups and $\$ 59$ for 3 groups with cluster grouping. These differences were even greater with OptiGroup, $\$ 48$ for 2 groups and $\$ 71$ for 3 groups. The OptiGroup outperformed (IOFC; $\$ /$ cow per yr) cluster by $\$ 8$ (range $\$ 6$ to $\$ 13$ ) with 2 groups and by $\$ 12$ (range $\$ 8$ to $\$ 17$ ) with 3 groups ( $P$ $<0.01$, Table 3). Without exception, IOFC increased with nutritional groups because of the reduced feed cost, whereas milk production remained the same. OptiGroup was always economically superior to cluster in each one of the 7 studied farms (Table 3). Results from cluster grouping are consistent with previous studies (St-Pierre and Thraen, 1999; Kalantari et al., 2016). Cabrera and Kalantari (2016) summarized previous studies and reported IOFC ( $\$ /$ cow per yr) between $\$ 21$ to $\$ 44$ for the 2-group scenario and $\$ 44$ to $\$ 46$ for the 3 -group scenario compared with the single-group scenario. Comparing between 2 grouping strategies, IOFC with cluster increased significantly from low to high group with the 2-group scenario and from low to medium to high with 3 -group scenarios $(P<0.01$, Table 4). OptiGroup IOFC was significantly greater in high group than low group in the 2 -group scenario $(P<0.01$, Table 4). In the 3-group scenario, OptiGroup IOFC was greatest in the medium group, followed by the high group with a significant difference between groups $(P<$ 0.01, Table 4). Although the OptiGroup medium group had the moderate feed efficiency (FE) among 3 groups and no significant lower feed cost comparing with the high group, the greater MY in the medium group led to the greatest IOFC (Table 4).

The present study explored a novel method of grouping cows, OptiGroup, that has an advantage over previous grouping criteria. We consider these results

Table 3. Income over feed $\operatorname{cost}^{1}$ (\$/cow per yr) for 1-group, 2-group, and 3-group scenarios, and difference of OptiGroup versus cluster

\begin{tabular}{|c|c|c|c|c|c|}
\hline \multirow[b]{2}{*}{ Farm } & \multirow[b]{2}{*}{ 1-group } & \multicolumn{2}{|c|}{ 2-group } & \multicolumn{2}{|c|}{ 3-group } \\
\hline & & Cluster & OptiGroup & Cluster & OptiGroup \\
\hline Farm 1 & 2,694 & 2,737 & 2,743 & 2,757 & 2,765 \\
\hline Farm 2 & 2,651 & 2,694 & 2,702 & 2,714 & 2,726 \\
\hline Farm 3 & 2,981 & 3,018 & 3,031 & 3,037 & 3,054 \\
\hline Farm 4 & 2,620 & 2,658 & 2,667 & 2,677 & 2,688 \\
\hline Farm 5 & 2,749 & 2,789 & 2,799 & 2,808 & 2,822 \\
\hline Farm 6 & 1,772 & 1,809 & 1,816 & 1,826 & 1,836 \\
\hline Farm 7 & 2,707 & 2,745 & 2,755 & 2,764 & 2,777 \\
\hline Average & $2,596^{\mathrm{a}}$ & $2,636^{\mathrm{b}}$ & $2,644^{\mathrm{c}}$ & $2,655^{\mathrm{d}}$ & $2,667^{\mathrm{e}}$ \\
\hline
\end{tabular}


Table 4. Nutrient requirement density, production performance, and profitability (mean \pm SD) of the 1-group scenario and each group with OptiGroup and cluster strategies with the 2- or 3-group scenarios

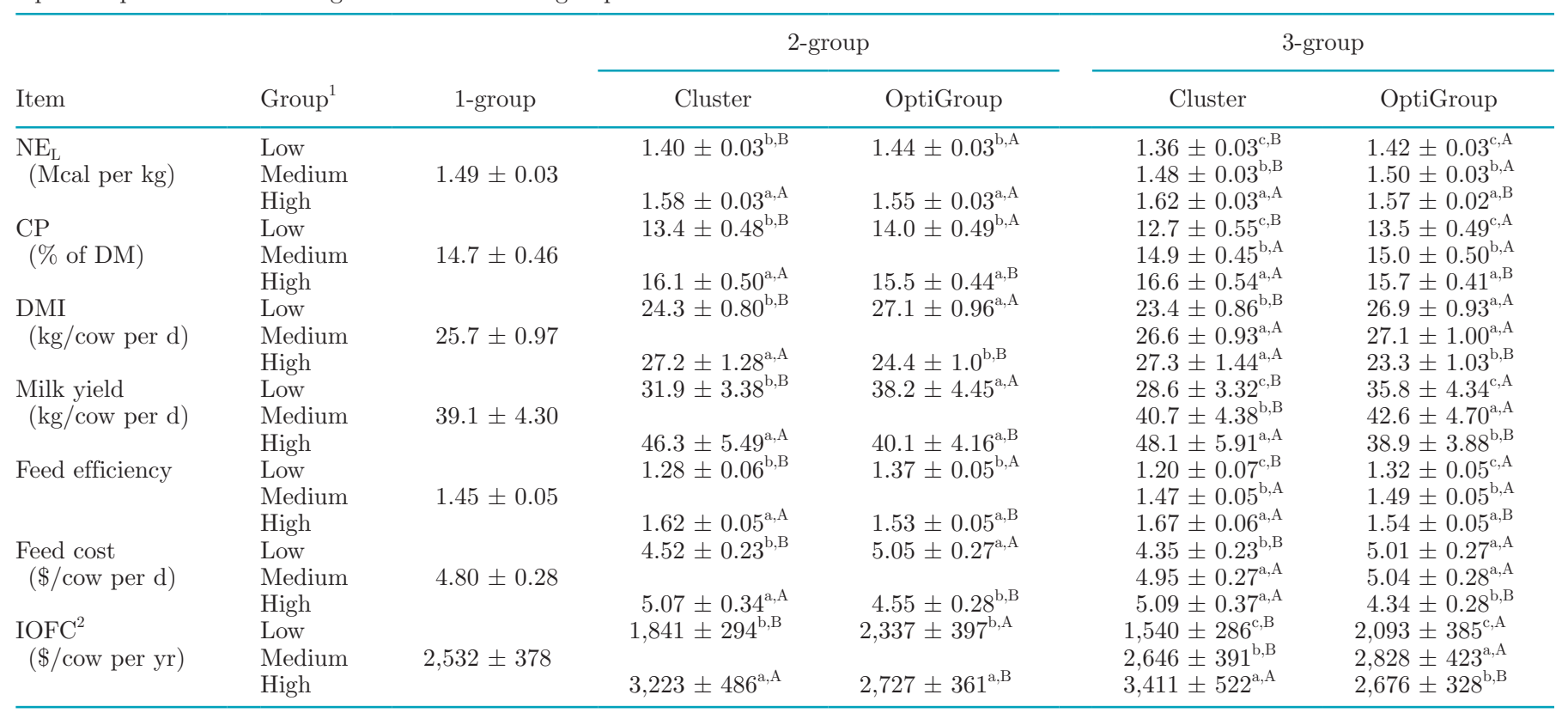

${ }^{\mathrm{a}-\mathrm{c}}$ Different lowercase letters represent a significant difference $(P<0.01)$ between groups within the same grouping strategy.

${ }^{\mathrm{A}, \mathrm{B}}$ Different uppercase letters represent a significant difference $(P<0.01)$ between OptiGroup and cluster strategies.

${ }^{1}$ Low, medium, and high groups refer to the nutrient requirement comparison: the high group had higher nutrient density than the medium group, and the medium group had higher nutrient density than the low group.

${ }^{2} \mathrm{IOFC}=$ income over feed costs.

a proof of concept that needs further validation with more secondary data and field trials. Nonetheless, we consider it to be a valid concept that should work under varied dairy herd conditions. If we consider cluster assumptions acceptable (Cabrera and Kalantari, 2016), OptiGroup is also acceptable and provides an advantage over cluster by assigning cows to groups within the optimization algorithm that always seeks the maximum IOFC. The cluster method groups cows a priori. We speculate that in the worst-case scenario, under a very rigid set of constraints, the OptiGroup will perform economically the same as the cluster method.

\section{Group Characteristics with Different Grouping Strategies}

Because cows were grouped differently with OptiGroup and cluster strategies, group characteristics were different between strategies. These results are listed in Table 4 under 2- or 3-group scenarios.

2-Group Scenario. With both strategies, the high group always had greater $\mathrm{NE}_{\mathrm{L}}$ and $\mathrm{CP}$ density, and feed efficiency than the low group $(P<0.01$, Table 4$)$. With cluster, DMI was significantly greater in the high group; however, the group-level DMI comparison was reversed with OptiGroup $(P<0.01$, Table 4$)$. With cluster, MY was significantly greater in the high group; however, the group-level MY had no significant difference between the OptiGroup high and low groups $(P$ $>0.05$, Table 4). The 2 groups with OptiGroup were more similar to each other in nutritional requirements and performances $\left(\mathrm{NE}_{\mathrm{L}}, \mathrm{CP}, \mathrm{DMI}, \mathrm{MY}\right.$, and $\left.\mathrm{FE}\right)$ than the 2 groups with cluster (Table 4 ).

3-Group Scenario. Cluster kept linear changes in cows' nutritional requirements and performance $\left(\mathrm{NE}_{\mathrm{L}}\right.$, $\mathrm{CP}, \mathrm{DMI}, \mathrm{MY}$, and FE) from the low to high group, with a significant difference between each 2 of the 3 groups $(P<0.01$, Table 4$)$, except no significant DMI difference between the high and medium group $(P>$ 0.05, Table 4). OptiGroup also showed a significant increasing in $\mathrm{NE}_{\mathrm{L}}$ and $\mathrm{CP}$ density requirement from low, medium, to high group $(P<0.01$, Table 4$)$. This was expected because the model constrained the high group to have the greatest nutritional requirements and the low group to have the lowest nutritional requirement. Different from the cluster groups, however, OptiGroup group-level MY followed nonlinear trends from low to high group. The MY was greatest in the medium group, followed by the high and low group with a significant difference $(P<0.01$, Table 4$)$. Group-level DMI was not different between low and medium group, whereas DMI in high group was significantly lower than both 
of the low and medium group DMI $(P<0.01$, Table 4). With OptiGroup, low group FE was significantly lower than the medium and high group, followed by the medium and high group with a significant difference $(P<0.01$, Table 4$)$. Same as the 2 -group scenario, the 3 groups with OptiGroup were more similar to each other in nutritional requirements and performances $\left(\mathrm{NE}_{\mathrm{L}}, \mathrm{CP}, \mathrm{DMI}, \mathrm{MY}\right.$, and $\left.\mathrm{FE}\right)$ than the 3 groups with cluster (Table 4).

\section{Dietary $N_{L}$ and CP Densities}

Adjusted by the lead factor, group-level dietary $\mathrm{NE}_{\mathrm{L}}$ and $\mathrm{CP}$ densities with different grouping strategies and number of ration groups are listed in Table 5 . The mean group-level dietary energy and protein densities were in the expected range of 1.44 to $1.72 \mathrm{Mcal} / \mathrm{kg}$ of $\mathrm{NE}_{\mathrm{L}}$ and 14.25 to $17.69 \%$ of CP for Holstein cows producing between 30 and $44 \mathrm{~kg} / \mathrm{d}$ (NRC, 2001). Note that the mean dietary $\mathrm{NE}_{\mathrm{L}}$ density for all cows decreased as the number of ration groups increased. The mean $\mathrm{NE}_{\mathrm{L}}$ density across groups decreased from $1.62 \mathrm{Mcal} / \mathrm{kg}$ for 1-group, to 1.58 with 2 -group, and to 1.56 for 3 -group. Dietary CP density (\% of daily DMI) decreased from $16.58 \%$ for 1-group to $16.02 \%$ for 2-group, and to $16.10 \%$ for 3-group (Table 5). In general, 1-group $\mathrm{NE}_{\mathrm{L}}$ and $\mathrm{CP}$ densities were in between the low and high group nutrient densities with 2-group scenario and in between the medium and high group nutrient densities with 3-group scenario. For example, with dietary $\mathrm{CP}$ density using the cluster method, average $\mathrm{CP}$ density with 1-group was $16.58 \%$, whereas 2 - and 3 -group were 14.87 and $17.16 \%$, and $14.25,15.44$, and $17.69 \%$, respectively (Table 5). The range of dietary $\mathrm{NE}_{\mathrm{L}}$ and $\mathrm{CP}$ densities with 2- and 3-group was narrower with OptiGroup than cluster (Table 5). For example, with dietary NE density (Mcal $/ \mathrm{kg}$ ) using the OptiGroup, the mean requirement decreased from 1.62 for 1-group to 1.58 for 2-group, and to 1.56 for 3-group. Herd-level feed cost per unit $(\$$ per $\mathrm{kg}$ of $\mathrm{DM}$ ) followed the same trend as the $\mathrm{NE}_{\mathrm{L}}$ and CP densities, decreasing from $\$ 0.179$ for the 1-group scenario to $\$ 0.174$ for OptiGroup and $\$ 0.175$ for cluster with the 2-group scenario, and to $\$ 0.172$ for OptiGroup and $\$ 0.175$ for cluster for the 3 -group scenario (data not shown).

\section{$N E_{L}$ and $C P$ Intakes}

Estimated average $\mathrm{NE}_{\mathrm{L}}$ intake of 40.06 to 41.62 Mcal/cow per d and average CP intake of 4.06 to 4.27 $\mathrm{kg} /$ cow per d (Figure 1) were within normal ranges for Holstein cows producing between 30 and $44 \mathrm{~kg} / \mathrm{d}$ (NRC, 2001). As expected and consistent with dietary nutrient densities above reported, estimated nutrient intakes (Mcal/cow per $\mathrm{d}$ and $\mathrm{kg} /$ cow per $\mathrm{d}$ ) decreased with increasing number of groups with either cluster or OptiGroup, and the decrease was greater for OptiGroup. For $\mathrm{NE}_{\mathrm{L}}$ (Mcal/cow per d, mean $\mathrm{NE}_{\mathrm{L}}$ across groups) the decrease was 0.83 for cluster and 1.06 for OptiGroup with 2-group and it was 1.28 for cluster and 1.57 for OptiGroup with 3-group. For CP (g/cow per d, mean CP across groups) the decrease was 130 for cluster and 150 for OptiGroup with 2-group, and it was 180 for cluster and 210 for OptiGroup with 3-group.

\section{Feed Cost}

On herd-level, feed cost determined the herd-level IOFC in both grouping strategies because milk production was assumed to remain constant. Lower herd-level feed cost resulted in higher IOFC. On group-level, feed cost depended on DMI and dietary nutritional densities; the latter one was always greatest in the high group and lowest in the low group with both grouping strategies. With the same grouping strategy, feed cost of each group was highly and positively related to DMI

Table 5. Average dietary nutrient densities for each group with both grouping strategies, with 2- or 3-group scenarios $^{1}$

\begin{tabular}{|c|c|c|c|c|c|}
\hline \multirow[b]{2}{*}{ Nutrient density by groups } & \multirow[b]{2}{*}{ 1-group } & \multicolumn{2}{|c|}{ 2-group } & \multicolumn{2}{|c|}{3 -group } \\
\hline & & Cluster & OptiGroup & Cluster & OptiGroup \\
\hline $\mathrm{NE}_{\text {low }}(\mathrm{Mcal} / \mathrm{kg}$ of DMI $)$ & - & 1.48 & 1.52 & 1.44 & 1.50 \\
\hline $\mathrm{NE}_{\text {medium }}(\mathrm{Mcal} / \mathrm{kg}$ of DMI $)$ & 1.62 & - & - & 1.52 & 1.53 \\
\hline $\mathrm{NE}_{\text {high }}($ Mcal $/ \mathrm{kg}$ of DMI $)$ & - & 1.68 & 1.63 & 1.72 & 1.66 \\
\hline $\mathrm{CP}_{\text {low }}(\%)$ & - & 14.87 & 15.54 & 14.25 & 15.13 \\
\hline $\mathrm{CP}_{\text {medium }}(\%)$ & 16.58 & - & - & 15.44 & 15.54 \\
\hline $\mathrm{CP}_{\text {high }}(\%)$ & - & 17.16 & 16.52 & 17.69 & 16.73 \\
\hline
\end{tabular}

${ }^{1}$ Group mean nutrient requirements are multiplied by lead factors (Table 2) to approximately satisfy nutritional requirements of $83 \%$ of cows of that group. $\mathrm{NE}=$ net energy. 
Cluster
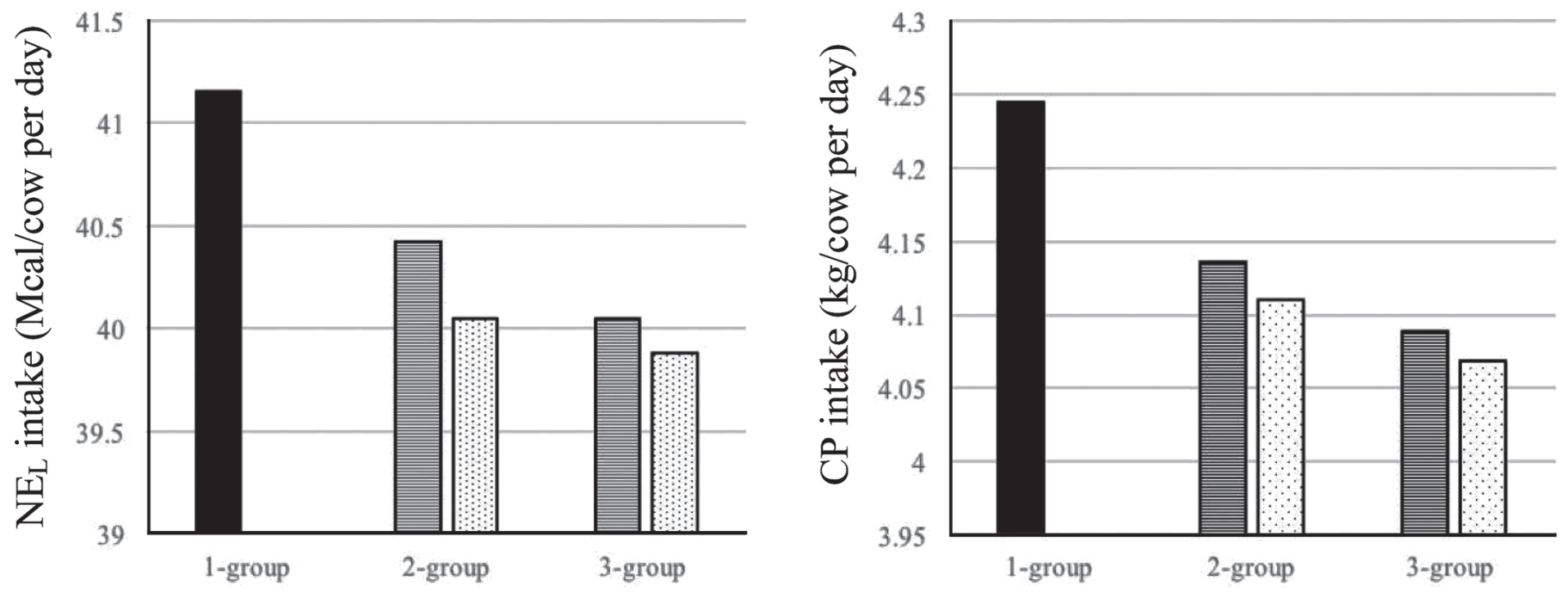

Figure 1. Estimated average energy $\left(\mathrm{NE}_{\mathrm{L}}\right)$ and protein $(\mathrm{CP})$ intake of the 7 studied farms.

(correlation $\geq 0.97$ ) for both 2 - or 3 -group scenarios (Table 4).

\section{Overall Performance}

The differences of cows' performance (MY, FE, DMI, $\mathrm{NE}_{\mathrm{L}}$, and $\mathrm{CP}$ ) and dietary nutrient densities were smaller in the OptiGroup compared with the cluster group. Comparing between grouping strategies, the OptiGroup low (and medium in the 3-group scenario) group had greater nutritional requirements than the counterparts in cluster; however, the OptiGroup high group had lower nutritional requirements than the counterpart in cluster. Reducing the difference of nutrient densities between diets could benefit dairy herd management with respect to grouping strategies. Farm managers would be more inclined to consider nutritional grouping if resulting diets are less drastically different than the original one. Cows would have less chance of suffering dietary stress caused by a dramatic change of diet when moved from one group to another group. The dietary stress caused by the large difference of dietary densities between groups may lead to over-feeding or negative balance. Over-feeding often results in over-conditioned cows, which are associated with shorter productive life, calving difficulties, metabolic diseases, or lower feed efficiency (Allen, 2009). Furthermore, excess CP intake also increases the manure $\mathrm{N}$ content, which leads to environmental concerns (VandeHaar and St-Pierre, 2006). Potential milk production decline caused by negative energy balance is also a concern expressed by dairy farm managers with respect to nutritional grouping
(Contreras-Govea et al., 2015). Trends and values from cluster NE and CP densities are very consistent with previous reports (McGilliard et al., 1983; St-Pierre and Thraen, 1999; Kalantari et al., 2016). Thus, we consider OptiGroup's nutrient density values and trends to be reasonable. The optimization algorithm sought the most economical allocation of cows to groups, which determined the final diets. Under the same assumptions of cluster and nutrient densities formulated at mean + $1 \mathrm{SD}$, the herd under OptiGroup would respond similarly to cluster because nutrient requirements of the groups' distribution are met (Stallings and McGilliard, 1984); however, with fewer overall nutrients provided, the farm could save feeding costs.

In the 2-step cluster grouping process, the model first grouped cows based on each cow's nutrient density requirement. In the second step, the model calculated diet nutrient densities for each group and calculated IOFC. Different from the cluster grouping, the OptiGroup strategy considered both of the cows' nutrient requirement and economic factors (milk, $\mathrm{NE}_{\mathrm{L}}$, and $\mathrm{CP}$ prices) simultaneously. Different from the cluster group that strictly allocated cows into groups only based on their nutritional requirements, OptiGroup separated cows with high nutritional requirement into different groups. With 3-group scenario, OptiGroup allocated the high-producing cows with moderate FE and nutritional requirements into the medium group and made the greatest IOFC than the other 2 groups. With lower dietary nutritional density in the high group, OptiGroup compensated the high group IOFC for the low and medium group IOFC and over-performed to cluster 
on herd-level IOFC (Table 4). The present study explored a novel method of grouping cows, OptiGroup, that outperformed the current state-of-art grouping criteria, the cluster.

A foundational assumption in grouping studies is that milk production remains the same after regrouping. As an approach to express cows' genetic potential, nutritional grouping optimally allocates feed resources (grain, forage, additives) to the cows to maximize feed efficiency and profitability (Allen and Piantoni, 2014; VandeHaar et al., 2016). Individual-cow level precision feeding through increased milk yield and composition and maintained the same DMI comparing with cows fed with one TMR diet (Maltz et al., 2013). Nutritional grouping also enables the nutritionist to alter the ration, such as change the fiber content or minerals, for a more focused cow group, which reduces the overall feed cost (VandeHaar et al., 2016). Considering the potential milk increase due to nutritional grouping, OptiGroup should have a greater advantage than cluster because the OptiGroup reflects milk production changes in grouping decisions.

\section{Cow Demographics in Each Group with Different Grouping Strategies}

Cows were grouped as having low and high nutrient requirements for the 2-group scenario, whereas cows were grouped as having low, medium, and high nutrient requirements for 3-group scenario. Allocation of cows in each group was not the same in cluster and OptiGroup. Of all 7 farms, 33.5 to $43.9 \%$ of cows with 2 groups and 54.7 to $75.1 \%$ of cows with 3 groups were grouped differently with OptiGroup compared with the cluster strategy (Figure 2). The OptiGroup created the groups integrally and interactively considering groups and their diet arrangements while maximizing IOFC. The cluster method first created the groups and then calculated the IOFC, which resulted in these differences. The percentage of cows grouped differently with 3 groups was higher than with 2 groups simply because there were more group alternatives for cows to be allocated. These results are neither surprising nor discouraging for OptiGroup.

Exploring more in depth the composition of the nutritional groups, we found that the percentage of primiparous cows in the 2-group for the high group was greater than in the low group with OptiGroup (54 vs. $22 \%$ of the group) opposite to the cluster (31 vs. $44 \%$ of the group; Figure 2A, left). For the 3-group, the percentage of primiparous cows increased from low to high groups with OptiGroup (32, 39, and 43\%), whereas more primiparous cows were allotted into the medium group with cluster ending with a proportion of primiparous cows of 40,47 , and $26 \%$ in the low, medium, and high groups, respectively (Figure 2A, right). The greater proportion of primiparous cows in the OptiGroup higher nutritional requirement group (high group in the 2-group scenario and medium and high group in the 3-group scenario) explained the lower DMI in those groups, because primiparous cows always have lower DMI due to the lower BW (NRC, 2001). ContrerasGovea et al. (2015) reported that some dairy farmers in Wisconsin and Michigan separate primiparous and multiparous cows. Although separating primiparous cows from the multiparous cows has a benefit on primiparous cows' social stress and productivity (Grant and Albright, 2001), allocating primiparous cows into an individual group is not the most profitable grouping option (Kalantari et al., 2016). The OptiGroup model allocated more primiparous cows into the same group and reached a greater IOFC than cluster strategy. If farms prefer to allocate primiparous cows into a separate group, it is yet expected that OptiGroup may reach a greater IOFC than cluster when grouping solely the multiparous cows.

Regarding the groups allocation of late-lactation cows (DIM > 200), the trend was similar for both cluster and OptiGroup (Figure 2B) with a greater proportion of these cows in the low group. With 2 groups, approximately 79 and $74 \%$ of cows in the low group were late-lactation cows for cluster and OptiGroup, respectively (Figure 2B, left). With 3 groups, majority of the late-lactation cows were in the low group $(85 \%$ of the group in cluster and $74 \%$ in OptiGroup were in late lactations). Cluster strategy allocated much more late-lactation cows in the medium group than the high group; however, the OptiGroup allocated a close number of late-lactation cows in groups with medium and high nutrient requirements.

\section{Sensitivity Analysis on IOFC}

With the 1-group scenario, increasing NE by $10 \%$ decreased the average farm-level IOFC by $\$ 106 /$ cow per year; increasing $\mathrm{CP}$ prices by $10 \%$ per $\mathrm{kg}$ decreased the average farm-level IOFC by $\$ 62.3 /$ cow per year. With the 2-group scenario, average farm-level IOFC across grouping strategies decreased $\$ 103.9$ for $10 \%$ NE price increase and $\$ 60.3$ for $10 \%$ CP price increase. With the 3-group scenario, average farm-level IOFC across 2 grouping strategies decreased $\$ 102.7$ for $10 \%$ $\mathrm{NE}_{\mathrm{L}}$ price increase and $\$ 59.5$ for $10 \% \mathrm{CP}$ price increase. Compared with the 1-group scenario, multiple groups are more resilient to the greater feed cost. In addition, the IOFC difference between a 1-group scenario and multiple group rations was slightly greater in the 3 -group scenario than 2-group scenario. Results from 
A.
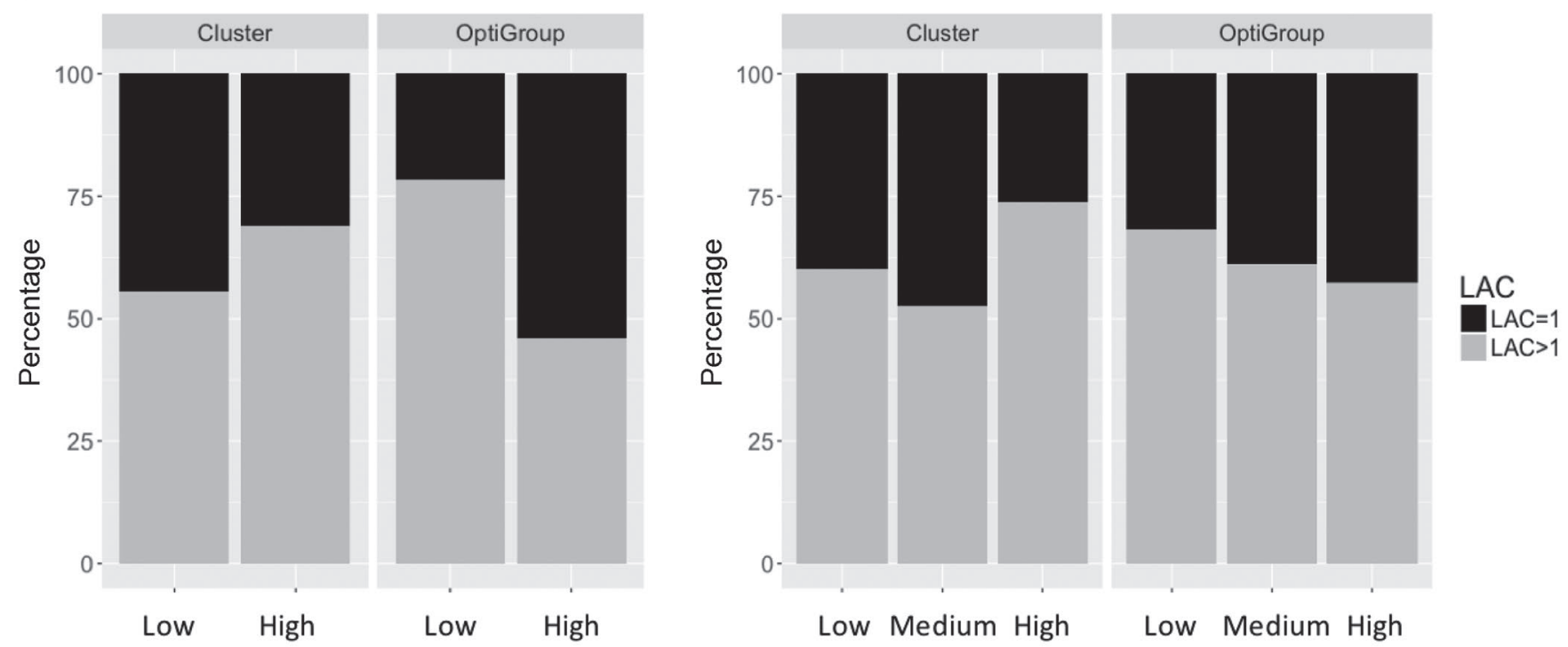

B.
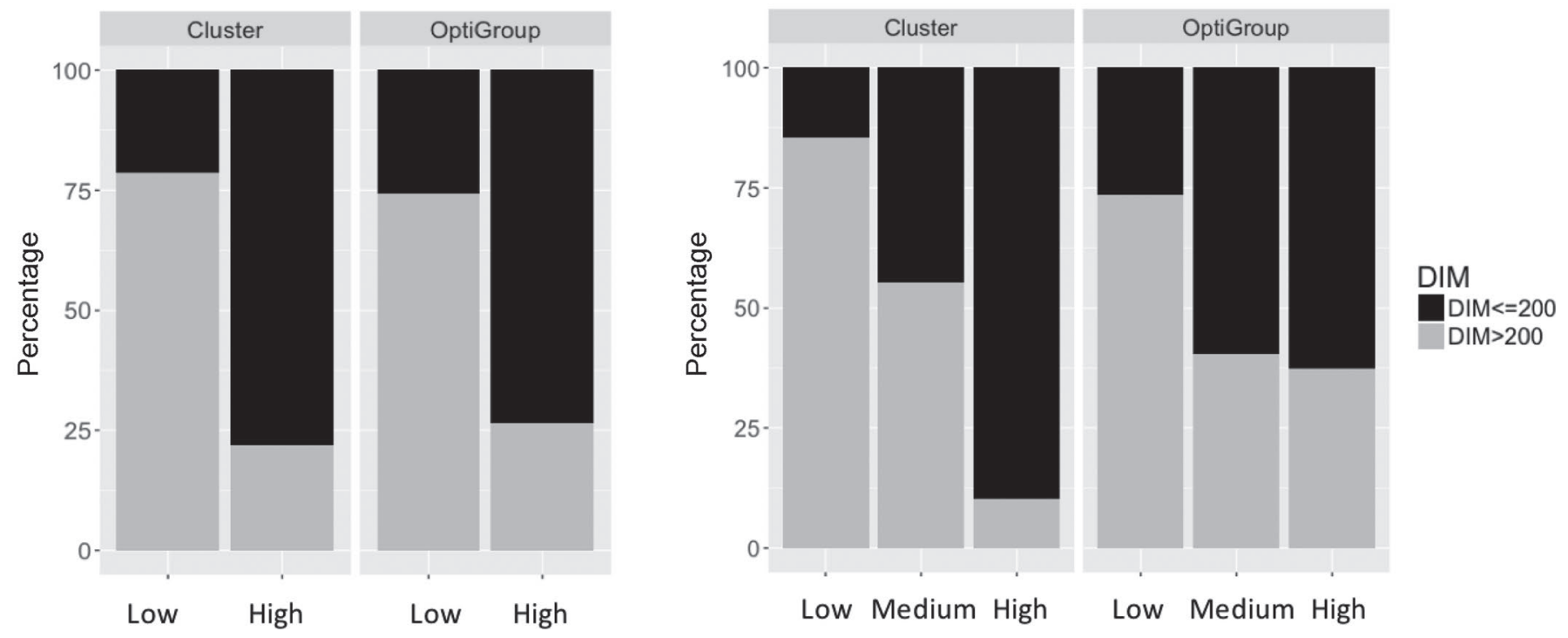

Figure 2. (A) Average percentage of primiparous cows $(\mathrm{LAC}=1)$ and multiparous cows (LAC >1). (B) Average percentage of early-lactation cows (DIM $\leq 200)$ and late-lactation cows (DIM >200) in the 2-group scenario (left) and the 3-group scenario (right) when using cluster and OptiGroup methods.

this project were in agreement with Kalantari et al. (2016) that reported the benefits of nutritional grouping compared with the 1-group ration under unfavored market conditions (high feed cost and low milk price). Although 3-group scenario showed higher IOFC than the 2-group scenario under all market conditions and grouping strategies, the trade-off between benefits and extra labor and facility costs of managing more groups needs to be considered in the decision-making process (Contreras-Govea et al., 2015; Kalantari et al., 2016).
With higher feed cost, OptiGroup always resulted in a greater IOFC than the cluster method in the 2- or 3 -group scenario. Taking the economic factors into the objective function also made the OptiGroup more sensitive to the market price changes. The IOFC advantage from the OptiGroup slightly enlarged as feed cost increased. In the 2-group scenario, increasing $\mathrm{NE}_{\mathrm{L}}$ price by $10 \%$ enlarged the difference between OptiGroup and cluster IOFC difference by $\$ 0.57 /$ cow per yr (from $\$ 8.92$ to $\$ 9.50 /$ cow per yr); increasing CP price by $10 \%$ 
enlarged the difference between OptiGroup and cluster IOFC difference by $\$ 0.32 /$ cow per yr (from $\$ 8.92$ to $\$ 9.24 /$ cow per yr).

It is clear that the optimization is seeking economic efficiency (OptiGroup) beyond the nutrient requirements (cluster) as it likely considers not only the nutrient density required with each permutation, but also the economic trade-off value. OptiGroup demonstrated an advantage over the cluster by assigning cows to groups within the optimization algorithm that always seeks the maximum IOFC.

\section{Limitations of Study}

Although OptiGroup performed better in farm-level IOFC than the cluster, it also requires greater computing power and advanced software to accomplish the algorithms. Even in our analysis, we needed to partially linearize the optimization algorithm to deal with the complexity of the model. Nonetheless, these issues would be resolved as technology improves and becomes even more available. Similar with all the other grouping strategies, OptiGroup is a one-time grouping strategy that does not project cows' future performance and group cows. As the quantitative effects of regrouping on milk production and feed intake are still unclear (von Keyserlingk et al., 2008; Schirmann et al., 2011; Zwald and Shaver, 2012), OptiGroup assumed no change in milk production and feed intake after moving cows. The OptiGroup model assumed the same sizes and same $\mathrm{CP}$ and $\mathrm{NE}_{\mathrm{L}}$ price across groups; however, the feeding group sizes and feed cost always vary on actual farms. The model is flexible to adjust to different group sizes and feed cost to reflect the realistic situations, but those were out of the scope of the present study. Future improvement on all the grouping strategies should aim for real-time cow regrouping and minimize the cow movements from one group to another group. Integrating cows' daily performance would also help monitor the social and production stress after regrouping, which can be further included in designing nutritional grouping strategies.

\section{CONCLUSIONS}

The proposed OptiGroup nutritional grouping method had better economic performance than the cluster method. The IOFC from OptiGroup was better than the cluster by $\$ 8 /$ cow per yr with 2 groups and by $\$ 12 /$ cow per yr with 3 groups. The reason for the higher OptiGroup IOFC was savings on feed costs by using less $\mathrm{NE}_{\mathrm{L}}$ and $\mathrm{CP}$ in the diet. Additionally, a smaller difference between dietary nutrient densities when using OptiGroup could be considered an advantage when moving cows from one group to another group. Group allocations were different between the 2 methods, and this difference became larger from 2-group scenario to 3 -group scenario. Primiparous cows appeared to account for a larger proportion of the low group when using the cluster method, but a smaller proportion when using the OptiGroup method. However, late-lactation cows always accounted for a larger proportion of the low group either using the cluster or OptiGroup method. In addition, OptiGroup performed better than the cluster under the high feed cost condition. OptiGroup considers market prices and cows' performance directly in the grouping decisions and diet formulation, which is more flexible to farm or market condition changes. Although we are confident in these results, more studies are required to validate them. These future studies should include field trials and a function of milk productivity change with diet changes.

\section{ACKNOWLEDGMENTS}

This research was supported by Hatch Project no. WIS01849 to V. E. Cabrera and R. D. Shaver.

\section{REFERENCES}

Allen, M. S. 2009. Grouping to increase milk yield and decrease feed costs. Pages 61-65 in Tri-State Dairy Nutr. Conf., Ft. Wayne, IN. The Ohio State University, Columbus.

Allen, M. S., and P. Piantoni. 2014. Carbohydrate nutrition: Managing energy intake and partitioning through lactation. Vet. Clin. North Am. Food Anim. Pract. 30:577-597. https://doi.org/10.1016/j .cvfa.2014.07.004.

Arora, J. S., M. W. Huang, and C. C. Hsieh. 1994. Methods for optimization of nonlinear problems with discrete variables: A review. Struct. Optim. 8:69-85. https://doi.org/10.1007/BF01743302.

Bussieck, M., and A. Meeraus. 2004. General algebraic modeling system (GAMS). Appl. Opt. 88:137-158. https://doi.org/10.1007/978 -1-4613-0215-5_8.

Bussieck, M. R., G. D. Corp, and P. Street. 1985. MINLPLib-A collection of test models for mixed-integer nonlinear programming. INFORMS J. Computing 15:1-119.

Cabrera, V. E., and A. S. Kalantari. 2016. Economics of production efficiency: Nutritional grouping of the lactating cow. J. Dairy Sci. 99:825-841. https://doi.org/10.3168/jds.2015-9846.

Carvalho, P. D., A. H. Souza, M. C. Amundson, K. S. Hackbart, M. J. Fuenzalida, M. M. Herlihy, H. Ayres, A. R. Dresch, L. M. Vieira, J. N. Guenther, R. R. Grummer, P. M. Fricke, R. D. Shaver, and M. C. Wiltbank. 2014. Relationships between fertility and postpartum changes in body condition and body weight in lactating dairy cows. J. Dairy Sci. 97:3666-3683. https://doi.org/10.3168/ jds.2013-7809.

Contreras-Govea, F. E., V. E. Cabrera, L. E. Armentano, R. D Shaver, P. M. Crump, D. K. Beede, and M. J. VandeHaar. 2015. Constraints for nutritional grouping in Wisconsin and Michigan dairy farms. J. Dairy Sci. 98:1336-1344. https://doi.org/10.3168/ jds.2014-8368.

Grant, R. J., and J. L. Albright. 2001. Effect of animal grouping on feeding behavior and intake of dairy cattle. J. Dairy Sci. 84:E156E163. https://doi.org/10.3168/jds.S0022-0302(01)70210-X.

Grossmann, I. E. 1990. Mixed-integer nonlinear programming techniques for the synthesis of engineering systems. Res. Eng. Des. 1:205-228. https://doi.org/10.1007/BF01581212. 
Holstein Association USA. 2018. History of the Holstein Breed. Accessed Feb. 4, 2018. http://www.holsteinusa.com/holstein_breed/ breedhistory.html.

Kalantari, A. S., L. E. Armentano, R. D. Shaver, and V. E. Cabrera. 2016. Economic impact of nutritional grouping in dairy herds. J. Dairy Sci. 99:1672-1692. https://doi.org/10.3168/jds.2015-9810.

Korver, S., J. M. van Arendonk, and W. J. Koops. 1985. A function for live-weight change between two calvings in dairy cattle. Anim. Prod. 40:233-241. https://doi.org/10.1017/S0003356100025332.

Maltz, E., L. F. Barbosa, P. Bueno, L. Scagion, K. Kaniyamattam, L. F. Greco, A. De Vries, and J. E. P. Santos. 2013. Effect of feeding according to energy balance on performance, nutrient excretion, and feeding behavior of early lactation dairy cows. J. Dairy Sci. 96:5249-5266. https://doi.org/10.3168/jds.2013-6549.

McGilliard, M. L., J. M. Swisher, and R. E. James. 1983. Grouping lactating cows by nutritional requirements for feeding. J. Dairy Sci. 66:1084-1093. https://doi.org/10.3168/jds.S0022-0302(83)81905 -5 .

NRC. 2001. Nutrient Requirements of Dairy Cattle. 7th rev. ed. The National Academies Press, Washington, DC.

R Core Team. 2017. R: A Language and Environment for Statistical Computing. R Found. Stat. Comput. Version 3:3503. https://doi .org/10.1007/978-3-540-74686-7.

Schirmann, K., N. Chapinal, D. M. Weary, W. Heuwieser, and M. A. G. Von Keyserlingk. 2011. Short-term effects of regrouping on behavior of prepartum dairy cows. J. Dairy Sci. 94:2312-2319. https: //doi.org/10.3168/jds.2010-3639.
St-Pierre, N. R., and C. S. Thraen. 1999. Animal grouping strategies, sources of variation, and economic factors affecting nutrient balance on dairy farms. J. Anim. Sci. 77(Suppl 2):72-83.

Stallings, C. C., and M. L. McGilliard. 1984. Lead factors for total mixed ration formulation. J. Dairy Sci. 67:902-907. https://doi .org/10.3168/jds.S0022-0302(84)81386-7.

VandeHaar, M. J., L. E. Armentano, K. Weigel, D. M. Spurlock, R. J. Tempelman, and R. Veerkamp. 2016. Harnessing the genetics of the modern dairy cow to continue improvements in feed efficiency. J. Dairy Sci. 99:4941-4954. https://doi.org/10.3168/jds .2015-10352.

VandeHaar, M. J., and N. St-Pierre. 2006. Major advances in nutrition: Relevance to the sustainability of the dairy industry. J. Dairy Sci. 89:1280-1291. https://doi.org/10.3168/jds.S0022 -0302(06)72196-8.

von Keyserlingk, M. A., D. Olenick, and D. M. Weary. 2008. Acute behavioral effects of regrouping dairy cows. J. Dairy Sci. 91:10111016. https://doi.org/10.3168/jds.2007-0532.

Williams, C. B., and P. A. Oltenacu. 1992. Evaluation of criteria used to group lactating cows using a dairy production model. J. Dairy Sci. 75:155-160. https://doi.org/10.3168/jds.S0022-0302(92)77749 -2 .

Zwald, A., and R. D. Shaver. 2012. Case study: Effect of pen change on milk yield by dairy cows in 2 commercial herds. Prof. Anim. Sci. 28:569-572. https://doi.org/10.15232/S1080-7446(15)30407-1. 